\title{
UN MODELO DE CONSEJERÍA GRUPAL PARA ESTUDIANTES IMPACTADAS POR LA VIOLENCIA
}

\section{GROUP COUNSELING FOR DATING VIOLENCE}

\author{
Ángel Villafañe Santiago* \\ María I. Jiménez Chafey** \\ Manuel Capellas Casellas*** \\ Shirleen Collazo Santos*****
}

RESUMEN

Este artículo describe el desarrollo de un modelo de consejería grupal para trabajar con estudiantes universitarias en la Universidad de Puerto Rico que han experimentado violencia en su relación de pareja. Los objetivos fueron: (1) reflexionar y conceptualizar el fenómeno de la violencia en el noviazgo; (2) aportar sugerencias para la creación de un modelo intervención grupal; (3) iniciar el modelo de consejería grupal enfocado en el apoderamiento $y$ destrezas de manejo; $y$ (4) asumir la responsabilidad en la formación integral en cuanto a la equidad de género, la justicia y el respeto a los derechos humanos. Luego se presentan datos sobre el impacto y eficacia del modelo recopilados a través de un estudio cualitativo donde se realizaron entrevistas semi-estructuradas a cuatro jóvenes que participaron del mismo demostrando que el modelo ayudó en los aspectos personales y de relación de pareja de las participantes.

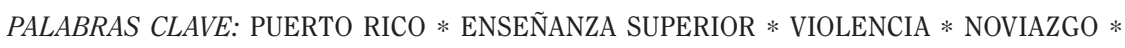

* Departamento de Consejería para el Desarrollo Estudiantil de la Universidad de Puerto Rico, Recinto de Río Piedras.

angelpr39@gmail.com

** Departamento de Consejería para el Desarrollo Estudiantil de la Universidad de Puerto Rico, Recinto de Río Piedras. maria.jimenez16@upr.edu
*** Docente, Universidad Politécnica de Puerto Rico. capella.manuel@gmail.com

**** Departamento de Consejería para el Desarrollo Estudiantil, Universidad de Puerto Rico, Recinto de Río Piedras.

shirleenc_4008@yahoo.com 


\section{ABSTRACT}

The first part of this article describes the process of starting a counseling group for female university students who have experienced dating violence in their relationships and the resulting model that emerged from the process of developing the group. The objectives were to: (1) reflect and conceptualize the phenomenon of dating violence, (2) make suggestions for the creation of a group intervention model, (3) initiate a group intervention focused on empowerment and coping skills and (4) assume responsibility in the integral formation related to gender equality, justice and respect for human rights. Lastly, data on the impact and efficacy of the group counseling model collected through a qualitative study are presented. This study consisted of semi-structured interviews conducted with four of the participants and the results demonstrate that the group helped with both personal and relationship issues in the participants.

\section{KEYWORDS: PUERTO RICO * HIGHER EDUCATION * VIOLENCE * ENGAGEMENT *} COLLEGE STUDENTS * WOMEN

La violencia en las parejas es un problema a nivel mundial. La UNICEF (2000) alertó sobre la violencia doméstica y documentó que un número creciente de investigaciones confirman la difusión de la violencia en todas las partes del mundo. Se calcula que entre el $20 \%$ y $50 \%$ de las mujeres han tenido experiencias de violencia doméstica, con ciertas variaciones entre países. Por su parte, el Center for Disease Control (2007) de los Estados Unidos proclamó a la violencia doméstica como un grave problema de salud pública que afecta a más de 32 millones de norteamericanos incluyendo a parejas adultas y jóvenes. Asimismo, la Organización Mundial de la Salud (2005) alertó que entre una cuarta parte y la mitad de esta población ha sufrido lesiones físicas por parte de su pareja lo cual sugiere que está más expuesta a la violencia en el hogar.

A escala mundial, la violencia entre las parejas adolescentes $y$ de jóvenes muestran niveles de riesgo y peligro de una magnitud superior a la de las parejas adultas (Jackson, Cram y Seymour, 2000). De igual forma, Kury, Obergfell, Fuchs y Woessner (2004) señalaron que las jóvenes son objeto de más ataques violentos que las mujeres de mayor edad. Echeburúa, FernándezMontalvo y Corral (2008) encontraron que tanto los agresores como las víctimas tienden a ser jóvenes, particularmente entre los 18 y 30 años. Por otro lado, estudios con parejas de adolescentes gay y lesbianas reportaron que aproximada- mente una cuarta parte habían experimentado violencia en su relación (Halpern, Young, Waller, Martin y Kupper, 2004).

La revisión de literatura arroja índices altos de violencia entre parejas en estudiantes universitarios en diferentes países. En los Estados Unidos, el National College Health Assessment reveló que de una muestra de 11408 estudiantes de diferentes universidades, el 12,1\% habían tenido relaciones emocionalmente abusivas (American College Health Association, 2006). En España, Muñoz Rivas (2006) encontró que el 45\% de las estudiantes de la muestra del estudio señaló que sus parejas les habían realizado algún comentario para molestarlas cuando mantenían una discusión, el 18,8\% fueron insultadas y el 2,7\% recibieron amenazas de agresión física. En México, se evidencian altos niveles de violencia en las relaciones de pareja en jóvenes, con prevalencias que fluctúan entre el 17 y 28\% (Rivera-Rivera, Allen, Rodríguez Ortega, Chávez Ayala y Lazcano-Ponce, 2006). En Venezuela, Rodríguez (2007) realizó un estudio sobre los casos atendidos en terapia de pareja en la universidad y encontró que $62 \%$ fueron por relaciones violentas en el noviazgo.

En Puerto Rico hay una escasez de estudios relacionados al tema de la violencia en las parejas en jóvenes universitarios. Se destaca un estudio realizado por Guenard (1998) donde se exploró la experiencia de violencia sexual 
y física a través de una encuesta a 208 estudiantes universitarios en Puerto Rico. El 6,8\% indicó haber sido víctima de abuso sexual en el noviazgo y el 8,5\% expresó que su pareja le había presionado para que sostuviera relaciones sexuales como una evidencia de compromiso entre ellos.

Las investigaciones revisadas relacionadas con la violencia han señalado la magnitud del problema, los factores asociados a ella $y$ el impacto en niños, jóvenes $y$ adultos con sus repercusiones en las relaciones de pareja cuando llegan a la adultez. En la población universitaria provoca además, un impacto directo en el área académica y vocacional. Siendo esto así, fue necesario realizar un análisis del problema de manera estructurada y sistemática que proveyera información para el desarrollo de estrategias de intervención. De esta manera se comenzó el proceso de formar un grupo de consejería para mujeres impactadas por la violencia para julio de 2007. A la par con el ofrecimiento de consejería individual a los estudiantes que solicitaban servicios en el Departamento de Consejería para el Desarrollo Estudiantil (DCODE), de la Universidad de Puerto Rico, se realizó un estudio en acción sobre la cantidad de mujeres jóvenes que llegaban con problemas en la relación de parejas. Esto permitió la creación de una comunidad de aprendizaje en la que los compañeros de trabajo (consejeros profesionales, consejeros en rehabilitación, psicólogos y trabajadores sociales) discutían los casos de forma integral y estudiaban estrategias de intervención. Particularmente, se interesó en desarrollar una estrategia de consejería en la cual las jóvenes se pudieran beneficiar de las experiencias de otras en la solución de problemas. Esto obedeció a que los profesionales de ayuda estaban ofreciendo consejería individual a jóvenes féminas con esta problemática que provenían de referidos realizados por los profesores, profesionales de la conducta de la universidad y de los Proctors (líderes de pisos) de las residencias universitarias.

De un estudio exploratorio sobre las razones principales por las que los estudiantes universitarios solicitan servicios de consejería sobresalieron que la relación de pareja estaba entre las tres principales dificultades y las situaciones de violencia doméstica fluctuaban entre 3 y 44 por año (Jiménez y Villafañe, 2009). Como parte del análisis, se estudió la diversidad de referidos que llegaban de las diferentes entidades de la universidad, la frecuencia del problema de violencia y su impacto en el estado emocional y el rendimiento académico de las estudiantes. Todos estos aspectos condujeron al desarrollo de un modelo de consejería grupal como una alternativa de comunidad de aprendizaje para manejar el problema de la violencia en la relación de pareja en jóvenes universitarias.

\section{MODELO DE CONSEJERÍA GRUPAL PARA ESTUDIANTES IMPACTADAS POR LA VIOLENCIA EN LA RELACIÓN DE PAREJA}

Investigadores que han trabajado con violencia de pareja $y$ han diseñado programas de intervención para manejar este tipo de situación, afirman que la intervención grupal es muy efectiva. Por ejemplo, Lizardi y Molina (2003) expusieron que uno de los beneficios del proceso de consejería es que la intervención grupal puede ofrecerse a un mayor número de personas. Resaltan el hecho de que los miembros del grupo sirven de redes de apoyo entre sí. Todos los participantes del grupo tienen la oportunidad de exponer sus puntos de vista o experiencias vividas, lo cual fortalece la intervención del profesional. Por su parte, Matud, Gutiérrez y Padilla (2004) indicaron que el modelo de consejería grupal les da a los participantes la posibilidad de aprender distintas estrategias y de desdramatizar su propia situación, ayudándoles a comprender que no es un problema individual, que no son las responsables de la situación vivida y que las mismas pueden ser superadas.

En un estudio realizado con una metodología grupal para trabajar con víctimas de violencia, Lizardi (2002) encontró cambios positivos en las participantes (Lizardi y Molina, 2003). La mayoría mostraron un aumento en el nivel de autoestima, un cambio hacia una visión menos tradicional de los roles de género $y$ un alto nivel de satisfacción con los servicios. También, el modelo grupal demostró ser eficaz en promover 
en las participantes el iniciar gestiones para estudios o empleo, romper con el ciclo de la violencia doméstica, aumento de conocimiento sobre la violencia doméstica y sobre recursos de la comunidad.

Por su parte, Matud (2004) realizó una investigación para evaluar la efectividad de la terapia grupal con una muestra de 50 mujeres maltratadas por su pareja. Los resultados mostraron que las mujeres experimentaron una reducción significativa en su sintomatología de estrés postraumático, depresión, ansiedad y síntomas somáticos, aumentando su autoestima, confianza en sí mismas y el control de sus vidas. Aunque el análisis de la eficacia a largo plazo sólo se realizó con doce mujeres, se halló que la mejoría se mantiene a los doce meses tras el tratamiento.

Por otro lado, Fritch y Lynch (2008) examinaron distintas investigaciones $y$ hallazgos empíricos relevantes de terapia grupal y los modelos teóricos utilizados en el tratamiento de adultos sobrevivientes de violencia interpersonal en la pareja $y$ encontraron que hay evidencia consistente de los efectos positivos del tratamiento grupal. No obstante, los autores subrayaron que es necesario desarrollar más investigaciones para identificar los beneficios potenciales de la terapia grupal para sobrevivientes de violencia interpersonal en la pareja.

Por lo anterior, se decidió desarrollar un modelo de consejería grupal para mujeres impactadas por la violencia. El objetivo principal fue que las participantes se apoderaran de sus asuntos personales, académicos y sociales para mejorar su calidad de vida. Para lograr esto, se utilizaron los hallazgos de diferentes estudios sobre intervenciones para la violencia entre parejas, las técnicas de diferentes marcos teóricos aplicables a este problema social y de salud, la experiencia clínica con esta población y las estrategias desarrolladas por las mismas participantes durante el proceso grupal. Este artículo presenta el desarrollo del modelo y el proceso de grupo así como un estudio cualitativo dirigido a obtener datos relevantes sobre la satisfacción de las participantes y el impacto de la consejería grupal en sus vidas.

\section{DESARROLLO DEL GRUPO}

La población de estudiantes universitarios se encuentra en una etapa del ciclo evolutivo donde la relación de pareja es primordial para la formación de su identidad (Bordignon, 2005). Tomando esto en consideración, antes de conformarse el grupo, en la consejería individual, se definieron $y$ analizaron los elementos significativos de la relación en pareja, la violencia en las relaciones y las expectativas que se tienen en la convivencia compartida. Se realizó una revisión de literatura y una reflexión crítica sobre las necesidades y temas más relevantes a trabajar en el proceso grupal con esta población.

Dado lo anterior, se hizo necesario delinear unos objetivos específicos que permitieran la ponderación, el análisis y la intervención de la consejería grupal con estas jóvenes. Estos objetivos sirvieron de guía al modelo y de orientación a las participantes sobre su colaboración. Previo al inicio del grupo, durante el proceso de entrevista inicial o en el proceso de consejería individual las participantes fueron motivadas a: (1) reflexionar y conceptualizar el fenómeno de la violencia en el noviazgo; (2) aportar sugerencias para la creación de un modelo como una intervención novedosa y eficaz; (3) iniciar el modelo de consejería grupal enfocado en el apoderamiento y manejo adecuado de sus vidas; $y$ (4) asumir la responsabilidad en la formación integral en cuanto a la equidad de género, la justicia y el respeto a los derechos humanos. El Modelo de consejería grupal para mujeres impactadas por la violencia se desarrolló por fases $y$ etapas evolutivas, propias de uno previamente desarrollado desde la acción del ofrecimiento del servicio de consejería. De la reflexión sobre la acción, se identificaron tres fases: (1) Promoción, reclutamiento y retención; (2) Composición del grupo y (3) la Intervención grupal. A su vez, la intervención grupal se comprendió como un proceso por etapas que se describe en una visión panorámica del proceso grupal. 
FIGURA 1

ETAPAS DEL PROCESO EN EL GRUPO

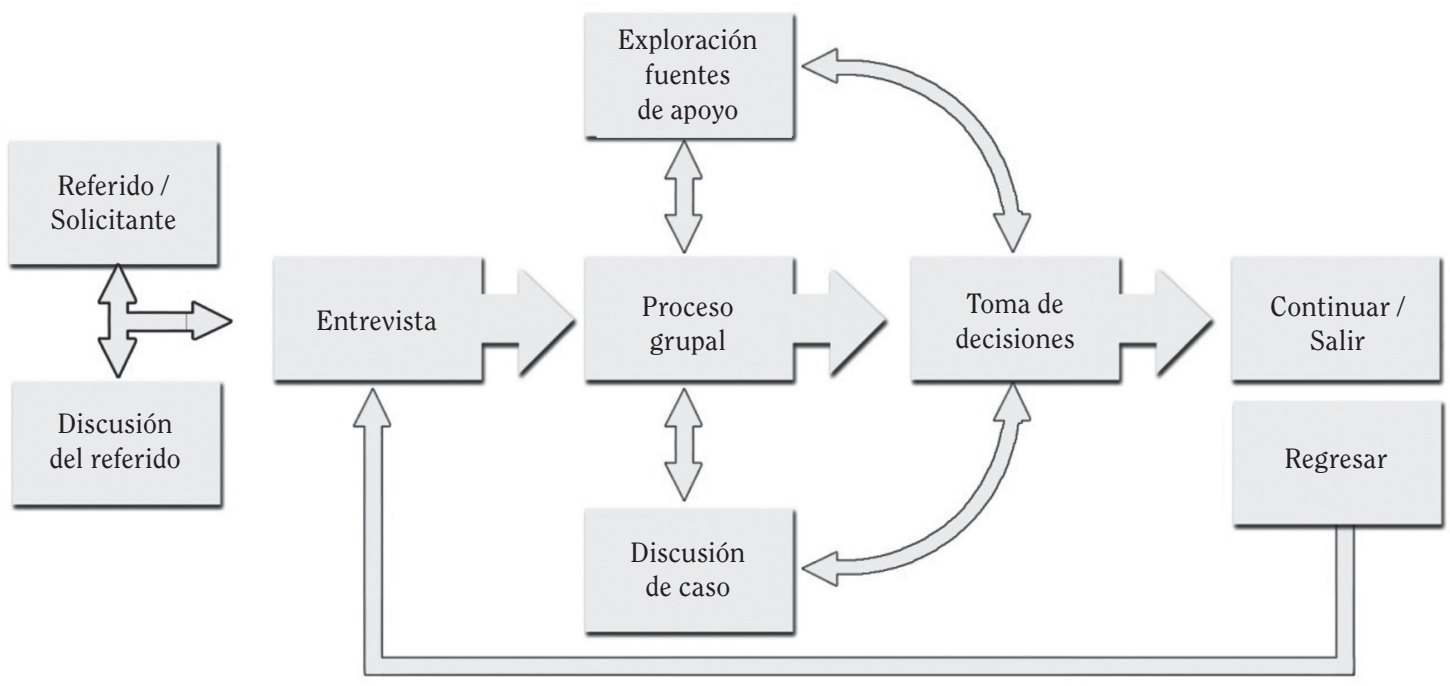

FASE 1: PROMOCIÓN, RECLUTAMIENTO Y RETENCIÓN. Se utilizaron diversas modalidades para hacer la promoción del grupo. Por ejemplo, se envío una hoja de promoción por medios electrónicos a los diferentes profesionales de ayuda en la universidad y a los estudiantes a través de hojas sueltas. Desde el plano profesional, se promocionó a través de conversaciones con profesores y colegas. La hoja de promoción se diseñó de modo que no subrayara la violencia en la relación de parejas como motivo del grupo. Esta acción obedeció a que muchas parejas no reconocen las diferentes situaciones relacionadas a la violencia y a su vez no pueden entender, aceptar o percibirlo como un problema grave.

Este modelo está centrado en el estudiante y su interacción con las otras participantes determina la funcionalidad del grupo. Por ello, se estableció un proceso de iniciación y continuidad. Las participantes entraron al grupo por diferentes vías. Por ejemplo, ellas podían haber llamado al facilitador (según la hoja de promoción) para una entrevista inicial en donde se le orientaría sobre el grupo. También, se recibieron participantes referidos de otros profesionales de ayuda, por lo que se realizaba una discusión del referido con el profesional. Si no fue un profesional de ayuda el que refirió entonces se procedía con la entrevista individual, sin la discusión del caso para proteger la confidencialidad de la estudiante. La entrevista permitió un proceso de empatía donde se exploraron los aspectos relacionados a la situación de violencia $y$ se discutió el proceso grupal. Con aquellas jóvenes que llegaron luego de haber comenzado el grupo, se aprovechó la entrevista inicial para explicar las áreas que se habían discutido, de manera que la persona asistiera a la próxima reunión orientada y pudiese participar de una manera más placentera e informada.

En la primera sesión se acordó el lugar de reunión, la duración y las reglas a seguir, incluyendo los aspectos de privacidad y confidencialidad. Para lograr la mayor asistencia, se les enviaba un mensaje electrónico que las llevara a reflexionar durante la semana y el recordatorio de la próxima reunión. También, se les llamaba vía telefónica el día antes y el mismo día de la reunión. Esto propició que las jóvenes fueran creando un sentido de pertenencia y percibieran que tenían disponible a un profesional de apoyo que les estaba ayudando en todo el proceso académico y personal.

FASE 2: COMPOSICIÓN Y ESTRUCTURA DEL GRUPO. El número máximo de participantes para el grupo fue de diez (10) lo cual se considera como un número óptimo para ser efectiva 
la consejería grupal por la complejidad de las situaciones que se pueden trabajar en el mismo (Yalom, 1985). La asistencia fluctuó entre cuatro a ocho participantes, con un promedio de cinco por sesión. Una de las decisiones emergentes de la dinámica del grupo fue que el mismo se mantuviera abierto, esto fue, que aquella participante que entendía que tenía las herramientas suficientes para manejar sus emociones y su proceso de vida, podía salir o volver a entrar sin estar atada a una estructura rígida. Esto permitió que otras jóvenes participaran del grupo sin tener que esperar un ciclo de cierre, obligando al grupo a atender las necesidades rápidamente. De esa manera, las participantes que entraron en diferentes etapas en su proceso de crecimiento pudieron servir de apoyo, e incluso de modelo.

Este primer grupo fue dirigido por un facilitador durante el primer año. No obstante, por lo intenso del trabajo se recomienda que sean dos co-facilitadores y que se roten cada año de manera que haya un periodo de descanso, evitando así el síndrome de quemazón que puede resultar del trabajo con grupos con problemáticas complejas. El proceso grupal se llevó a cabo en un salón con espacio adecuado para el número de participantes garantizando la confidencialidad de lo discutido. Los materiales básicos utilizados fueron: pizarra, marcadores, fotocopias de lecturas, presentaciones en PowerPoint, mensajes electrónicos, canciones y películas. Las sesiones duraban aproximadamente una hora y media, una vez a la semana.

FASE 3: INTERVENCIÓN GRUPAL. El modelo tiene unas estrategias o técnicas de diferentes marcos teóricos que fueron utilizadas para facilitar el proceso de intervención grupal, el manejo de los temas y las situaciones que presentan las jóvenes. Los más utilizados fueron: (a) teoría de la violencia transgeneracional (Blázquez Alonso, Moreno Manso y García-Baamonde, 2010), (b) teoría feminista (Barrón López, 2008), (c) teoría cognoscitivoconductual (Beck, Rush, Shaw y Emery, 1979), (d) teoría centrada en la persona (Rogers, 1997), y (e) teoría de la elección (Glasser, 1999). De igual forma, surgieron estrategias de manejo desarrolladas por las mismas participantes que contribuyeron tanto a su crecimiento personal como del grupo.

En promedio las jóvenes participaron de 20 sesiones. Una vez comenzado el grupo, se observó que las jóvenes pasaron por diferentes fases que se describen a continuación.

Ventilación de experiencias (Sentimientos). Las jóvenes comenzaron a expresar las experiencias vividas pasadas y las presentes donde evocaron emociones o sentimientos como: coraje, dolor, angustia, desesperanza, conflicto, insatisfacción por la vida y desvalidez.

Evaluación de las vivencias. Esta es una etapa de reflexión y retroalimentación. Las participantes interpretaron su vida, resurgieron emociones y se hicieron recomendaciones. Aquí cada participante reconoció que desvalida sus emociones y su toma de decisiones permitiendo ser manipulada $y$ creando codependencia en el proceso de convivencia con su pareja. También crearon conciencia de que al momento de querer realizar una acción como salir a divertirse, vestirse o relacionarse con otras personas solían pedir permiso a su pareja y no lo decidían por ellas mismas. Una de las estrategias que utilizaron las jóvenes al momento de la culminación de su relación de pareja es el "llevar la procesión por dentro" o el momento del duelo. Comienza con la etapa del duelo, y ellas expresan al grupo algunas conductas como encerrarse en sus cuartos a llorar el dolor que causa la separación, pero manteniendo la esperanza de que su pareja regrese.

Luego, se realiza un proceso de análisis de la situación momentánea. Durante esta fase, las participantes atraviesan por un proceso de desesperanza por volver con su pareja, pero que unido a una catarsis de llanto, van tomando decisiones. Por ejemplo, al llevar su "procesión", ellas comenzaron a incorporar nueva vestimenta, cambios de maquillaje $y$ peinados tratando de sentirse bien con ellas mismas. Como consecuencia, comenzaron a tener nuevas salidas sociales y de diversión.

Aprendizaje compartido. Las jóvenes empezaron a comunicar su aprendizaje en el grupo, ocurriendo una sinergesis al interaccionar las experiencias escuchadas de las otras participantes con las suyas. Para que esto sucediera, 
se dio un proceso de empatía previo. La identificación con el dolor de las otras le permitió ofrecer apoyo sanador. A su vez, usaron la resilencia como un proceso de fortalecimiento que las condujo a un estado de trascendencia que las habilitó para ayudar a las demás.

Adquisición de nuevo conocimiento. A través de este proceso de aprendizaje se pretendió que las participantes demostraran un cambio en actitud mientras procesaban información relacionada a: auto-concepto, autoestima, manejo de la soledad y el miedo, timidez, codependencia, autovalidación, modelo de las tres $R$ : responsabilidad por sus acciones, respeto a sí mismas y respeto a los otros (Navarro, 2008), la verdad, el ciclo de violencia (Bonilla, 2002), la rueda del poder y control (Partnership Against Domestic Violence, n.d.) y la rueda de equidad (Michigan Domestic Violence Prevention \& Treatment Board, n.d.), entre otras.

El despertar. Las jóvenes tomaron una nueva conciencia y comenzaron a verbalizar el reconocimiento del cambio que han tenido mientras fueron capaces de señalar aquellos temas que le ayudaron y reflexionaron sobre su proceso en el grupo.

Toma de decisiones. La capacitación grupal ayudó a las jóvenes a tomar decisiones viables o posibles, como por ejemplo, terminar la relación de pareja o continuar con la relación pero con nuevos términos saludables al bien individual y común. Por otro lado, algunas participantes se recuperaron de la pérdida de la pareja y decidían junto a su profesional de ayuda si continuaban en el grupo, en consejería individual o daban por culminados los servicios.

En este proceso, surgió el concepto del triángulo de la separación. Este triángulo se compone de: la separación, la distancia y la cognición. La separación es el acto de notificar que la relación ha concluido. La distancia es el romper con toda comunicación presencial o electrónica con la ex pareja o amigos y familiares que no estén ayudando en el proceso de manejo de la separación. La cognición constituye el aprendizaje de la experiencia de la relación de pareja $y$ del aprendizaje en el grupo para no caer en una relación similar.
Sistema de apoyo. Las participantes desarrollaron una red de apoyo que les fortaleció individualmente al ayudar a las otras. En esta etapa es donde se reconoce ampliamente a la consejería individual $y$ la grupal como parte importante en el proceso personal, académico y social.

\section{ESTRATEGIAS UTILIZADAS POR EL FACILITADOR}

Una de las estrategias principales fue la empatía, basada en la teoría centrada en la persona de Carl Rogers. Esta es utilizada tanto por el facilitador como las jóvenes con el objetivo de crear un ambiente de cohesión grupal donde las situaciones, emociones y conflictos son trabajados con respeto, apoyo y sensibilidad. Como mecanismo, este puede ser visto a través de un diagrama de Venn en que cuatro procesos interactúan (escuchar, analizar, debatir y contribuir) y para crear la zona de solidaridad o empatía. Paralelo con la anterior, en la consejería de pares las mismas jóvenes expresan $y$ escuchan, debaten $y$ entran a un proceso de reflexión, que luego les lleva a tomar decisiones sobre su relación de pareja.

Con la confrontación, debatían, sugerían y tomaban decisiones al respecto de la situación presentada al grupo. Se esperaba un comportamiento de estímulo-respuesta, donde la opinión debe ser argumentada e informada. Las asignaciones o tareas fueron cruciales como estrategia primeramente individuales. Algunas de ellas fueron: la discusión de un pensamiento enviado por un medio electrónico, la discusión de una lectura (Santiago, 1996) o ver alguna película. Estas actividades sirvieron de punto de partida para el desarrollo de la próxima sesión terapéutica. La auto-revelación se usó como un mecanismo para lograr empatía y confianza. Esto permitió que ellas reconocieran que hay otras personas (familiares, amigos o conocidos) que están viviendo la experiencia de violencia en la relación de pareja, y que no es un asunto exclusivo de ellas. En este sentido, fue un proceso reflexivo tanto para el facilitador como para las mismas participantes.

Este proceso grupal duró aproximadamente un año y medio lo que dio oportunidad a que estuvieran en constante crecimiento $y$ desarrollo. 
Es por eso que su estructura permite ver los elementos que dieron paso a su creación, las fases por las cuales fue pasando $y$ las estrategias en las intervenciones. Esto, en consecuencia permitió el desarrollo de un proceso de evaluación $y$ un estudio de investigación cualitativa donde se pudo ver la eficacia del trabajo realizado con las jóvenes.

\section{METODOLOGÍA DE EVALUACIÓN DEL MODELO Y SU IMPACTO}

La metodología de la investigación en acción es una propuesta de estudio, que por su carácter evolutivo es cambiante. Se desarrolló una evaluación general del grupo, tras un año y medio de intervención mediante el diseño de un estudio cualitativo con enfoque fenomenológico. Se escogieron, por disponibilidad, a cuatro participantes con el propósito de explorar el impacto de la consejería grupal. Las jóvenes estudiaban en la universidad y participaron del proceso de consejería grupal durante el 2008. Otros criterios de inclusión fueron: ser mayor de edad (21 años o más) $y$ haber participado en por lo menos ocho sesiones de consejería grupal.

La entrevista semi-estructurada, recogió en palabras de las participantes, un sentido de la amplitud y profundidad del impacto de la consejería grupal. La guía de entrevista contenía preguntas abiertas y cerradas dirigidas a recopilar información relacionada al impacto de la consejería grupal en las participantes. Incluía preguntas que recopilaban información sobre: datos socio-demográficos básicos de la participante, la relación de pareja que las llevó a participar del grupo, el impacto del grupo en su toma de decisiones y crecimiento personal, los elementos que fueron de mayor ayuda en el proceso y recomendaciones para mejorar el mismo.

\section{PROCEDIMIENTO}

Una vez que se obtuvo la aprobación del Comité Institucional para la Protección de Seres Humanos en la Investigación para llevar a cabo el estudio, se invitaron a las integrantes del grupo a participar de este. Todas las entrevistas fueron grabadas en audio y luego transcritas $y$ tuvieron una duración aproximada de entre cuarenta $y$ cinco minutos $y$ una hora $y$ media.

Participantes. Las edades de las participantes fluctuaron entre los 21 y 31 años. Al momento de su primera relación de pareja, la edad de ellas fluctuó entre los 13 y los 22 años. Tres de las participantes se encontraban en una relación de pareja, con una duración de entre 1 y 4 años. Dos de ellas se encontraban en la relación de pareja que motivó asistir al proceso de consejería, mientras que una tenía una relación de pareja no relacionada al motivo por el cual decidió participar de la consejería grupal. Para tres de las participantes el primer incidente de violencia se generó al inicio de la relación; una, recién casados y las otras dos, en el primer año de la relación.

\section{ANÁLISIS DE INFORMACIÓN}

Para el análisis de la información recopilada se siguieron las pautas de la teoría emergente (Strauss y Corbin, 1990; Luccas y Berríos, 2003). Las entrevistas transcritas se enumeraron del uno hasta el cuatro. La lectura de la entrevista nro.1 permitió establecer una primera codificación de las respuestas (codificación abierta). Una segunda lectura subrayó en posibles subcategorías. El mismo proceso se hizo con las otras entrevistas, para validar, añadir o debatir alguna categoría o proposiciones teóricas. Algunas de estas categorías fueron: el impacto de la consejería grupal, la toma de decisiones, el crecimiento personal. Del resumen de las entrevistas, se establecieron conexiones entre categorías (codificación axial). Se descartaron las respuestas que no guardaron relación alguna con el impacto del modelo. De esta codificación, se identificaron temas y patrones comunes. Como herramienta, se hizo una triangulación de viñetas extraídas de las entrevistas y los significados adjudicados por el grupo (Patton, 2002).

\section{HALLAZGOS}

El impacto de la intervención comenzó cuando las participantes reconocieron que 
habían estado expuestas a violencia en la relación de pareja y tomaron la decisión de permanecer en el grupo, con la intención de ayudarse y ayudar a las compañeras. Reconocieron la codependencia como un elemento negativo en las relaciones de pareja y modificaron patrones de interacción para "... prevenir cierta conducta "malsana" para la relación". De esa forma aumentaron su autoestima, la confianza en sí mismas y el control de sus vidas. El concepto de la universalidad que se da en los procesos de grupo como uno de los elementos terapéuticos fue resumido por una de las participantes:

- Me ha ayudado a conocer a otras personas que sufren o han sufrido lo mismo o algo parecido a lo que yo sufrí. Ahora puedo 'aconsejar' y ayudar a otras personas que están pasando por momentos difíciles.

Dentro de la categoría del impacto que tuvo la consejería grupal surgieron cinco subcategorías que fueron: (1) el manejo de las emociones, (2) la modificación de la conducta, (3) aumento en conocimiento, (4) valor propio y (5) toma de decisiones. En cuanto al manejo de las emociones, las participantes aprendieron a ventilar los sentimientos de forma positiva, a soltar y manejar las emociones fuertes como el coraje y el rencor,

-Poco a poco me di cuenta que no podía guardar esos rencores... no vale de nada tenerle un odio así.

- Me ha ayudado muchísimo en el manejo del coraje aunque todavía me falta mejorar completamente.

Esto reafirma que la recuperación de la pérdida de una relación es un proceso que conlleva tiempo, $y$ la experiencia de diferentes emociones en diversas intensidades ayuda a la persona a estar más receptiva para manejarlas:

-Ahora estoy más contenta porque ya he pasado el periodo de luto.
-Yo adquiría cosas buenas... cuando empecé, con mucha carga... y poco a poco sentía que iba soltando diferentes cosas... fui bajando, bajando, bajando... más estable, más tranquila.

Las participantes se dieron cuenta de cómo sus conductas contribuían a las interacciones tanto positivas como negativas dentro de las relaciones de pareja e incluso identificaron que estaban comenzando a modificar algunas conductas:

-Empecé a descubrir que en mi nueva relación... yo estaba manipulando.

—De cómo [otra compañera participante] tuvo que cambiar su temperamento para ser más sensible. Eso me ayudó porque me confirmó que uno siempre puede dialogar en vez de discutir y ser altanero.

-Yo veo cosas en la pareja que se parecen a mi papá y salgo bien explosiva. Ahora mismo sé que debo evitar esas cosas porque si yo quiero respeto también tengo que respetar.

Estos argumentos nos podrían estar indicando que las jóvenes con el problema de violencia en la relación de pareja avanzan en su proceso terapéutico cuando observan y escuchan experiencias de otras jóvenes que están pasando por la misma situación. También está relacionado a la comprensión humana descrita por Navarro (2008), como un concepto de doble intención que requiere conocer para comprender, y sólo se comprende si se conoce para apoyar $y$ para hacer crecer.

A su vez que reconocieron como su conducta afectaba las relaciones, también aumentó el conocimiento que tenían sobre ellas mismas $y$ sobre los elementos importantes que componen una relación saludable:

-Tomé conciencia de que uno tiende a repetir lo que la otra persona hace; también he recapacitado en cuanto a mis actitudes, de su valor propio. 
- Que uno debe valorarse y respetarse.

Aunque la persona le haya hecho daño a uno [hay] que respetarla también.

Por tal razón estas experiencias pueden ser relacionadas al desarrollo de la asertividad (Navarro, 2008), donde las participantes adquieren la habilidad para transmitir y recibir mensajes haciéndolo de forma honesta, profundamente respetuosa, directa y oportuna.

Partiendo del respeto a los demás y la responsabilidad por sus acciones según el modelo de Beauchamp, (1985) y citado por Navarro (2008) las jóvenes dieron valor al nuevo conocimiento que iban teniendo a medida que el grupo avanzaba. Ejemplo de ello lo podemos ver en las siguientes expresiones:

-El grupo me ayudó a ver [el asunto de] la responsabilidad. A veces pensé que todo era culpa de él,... otras mías. Ahora me doy cuenta que es miti-miti (sic).

- [En una relación amistosa reciente] empezó a mandar mensajes... y yo pude claramente identificar que es una forma de manipulación y de crear una codependencia conmigo.

-Yo me di cuenta de que él no iba a volver jamás en la vida y lo que me ayudó fue el escuchar todo lo que las muchachas dijeron. [Aprendí que] el no tener que aceptar todo [en una relación] y poder poner mis propias cláusulas también.

[En cuanto a la toma de decisiones, las participantes indicaron que el grupo les ayudó a tomar la decisión de terminar una relación dañina]:

—El divorcio... él no quiso venir para el acuerdo mutuo... acá yo empecé a mover el divorcio por separación, [al sentirse seguras de decisiones que habían tomado]. —Me ayudó a afirmar mi decisión [y a establecer límites saludables al terminar la relación]. - Esta vez [que me botó] le dije las cosas como eran, que me dejara tranquila, que me respetara y que no pensaba ir a su apartamento..., tuve que quitar Facebook... sacarlo de Messenger... cambiar mi teléfono... para poderlo separar.

Estas expresiones confirman el aprendizaje en la toma de decisiones y el uso de la estrategia del triángulo de la separación desarrollada durante el proceso grupal. La misma iba dirigida a que una vez que se lograra la separación, había que establecer la distancia (evitando toda comunicación con la ex pareja) y aprender del proceso para no volver a repetir la misma conducta en otras relaciones de pareja.

Las participantes reconocieron que la fase de instrucción del modelo fue muy útil, en particular los temas de autoestima y codependencia. Una evidencia del impacto del modelo en la modificación de conducta es el proceso de asimilar y adaptar nuevo vocabulario. Entre los temas de mayor impacto que mencionaron las participantes fueron:

-El respeto... de que uno debe valorarse a sí mismo.

-El ciclo de control y poder [para saber lidiar con las preferencias individuales y cómo considerarlas].

—El tema del perdón porque tengo muchos hilos sueltos (sic) con mi familia y los puedo llevar a mi relación... y lo del triángulo.

—El tema de la violencia en las parejas y al extremo que llegan $y$ presentaron un documental de esto.

Entre las recomendaciones que ofrecieron las participantes, se encontró que se le podría dar más énfasis a ciertos temas como "la soledad", "el manejo de la separación" y la "sexualidad", ya que sintieron que a veces no se podían trabajar con mayor profundidad por falta de tiempo:

-A veces nos quedamos cortos de tiempo, pues hay temas que se deberían discutir mucho mejor. 
- Me parece que un semestre es un tiempo muy corto para todo lo que hay que cubrir...

Otra participante comentó sobre el tamaño del grupo enfatizando que no debía excederse de 10 participantes

- Me sentía cada semana que estaba con un grupo diferente... pues al final empezaron a ir otras muchachas también.

Otra comentó sobre la importancia de la continuidad en la asistencia para aumentar la eficacia del proceso,

- yo trataba de ir a todas las reuniones... pero notaba que las otras no eran tan responsables... había que repetir la historia otra vez.

Las participantes mencionaron varias ventajas y elementos que les gustaron del grupo entre ellos algunos relacionados al facilitador, al material repartido y a la dinámica que se daba en el grupo. Una participante indicó que le fue muy útil el material escrito que se le fue entregado al final porque muchas veces se le olvidaban las cosas y le servía de repaso del material discutido. En cuando a la dinámica del grupo comentaron que se fomentaba mucho la interacción y la solidaridad, escuchando las cosas de las muchachas me ayuda a ver, me identifico. Entre las ventajas del grupo mencionaron el horario y el costo-efectividad del mismo:

- gracias a Dios que hay horario extendido porque sino no puedo participar,

- cualquier psicólogo te va a cobrar un chaval. [En cuanto al rol del consejero facilitador del grupo enfatizaron que sirvió de agente despertador]:

—Él nos abría los ojos... a cosas diferentes, a diferentes patrones. [También expresaron que fungió como guía y educador] - como que él nos trataba de confundir a propósito... para cogerlas; preguntas que nos quedábamos [en blanco].
Por ejemplo, ¿qué es la felicidad? Era como tratando de llevarnos poquito a poco.

$\mathrm{Al}$ analizar el proceso de consejería grupal que se ha presentado, se puede observar que es dinámico, progresivo y cambiante. Como estrategia, permitió identificar las distintas características de la manifestación de la violencia en la relación de parejas jóvenes universitarias $y$ a su vez les ayudó en su proceso rehabilitativo-habilitativo. Esto se dio en la medida que ellas pudieron identificar y aceptar la violencia como uno de los mayores factores que incidía en la estabilidad de la relación de pareja así como las repercusiones al nivel personal, social y académico. En términos de la implantación del modelo, sugiere además, que los profesionales de ayuda que deseen iniciarlo pueden modificarlo de acuerdo con la población que impactarán y al escenario donde se desarrolle. A esos efectos, podemos concluir que es una estrategia donde las personas pueden aprender, modificar y cambiar conductas para mejorar su proceso de vida $y$ ser más asertivas en su toma de decisiones.

\section{REFERENCIAS}

American College Health Association. "National College Health Assessment Spring 2006 Reference Group Data Report”. Journal of American College Health 55 (4). American College Health Association, 2007: 195-206.

Barrón López, Sara. "Investigación empírica y teoría feminista en los estudios familiares en el mundo anglosajón: una síntesis extramuros". Empiria: Revista de Metodología de Ciencias Sociales 15. Universidad Nacional de Educación a Distancia. Enero-junio 2008: 75-98.

Beck, Aaron T.; Rush, A. John; Shaw, Brian F. y Emery, Gary. Cognitive therapy of depression: A treatment manual. New York: Guilford Press, 1979. 
Blázquez Alonso, Macarena; Moreno Manso, Juan Manuel y García-Baamonde Sánchez, María Elena. "Revisión teórica del maltrato psicológico en la violencia conyugal". Psicología y Salud 20(1). Instituto de Investigaciones Psicológicas Universidad Veracruzana, 2010: 65-75.

Bonilla, Zobeida. "Guía de capacitación para promotoras de salud basada en el libro Nuestros cuerpos, nuestras vidas. Taller de capacitación: La violencia en contra de la mujer". Colectiva de Salud de las Mujeres de Boston. 2002. En: <http:// www.ourbodiesourselves.org/uploads/ pdf/violen.pdf> [Consultado: 2008].

Bordignon, Nelson Antonio. "El desarrollo psicosocial de Eric Erikson. El diagrama epigenético del adulto". Revista Lasallista de Investigación 2 (2). Corporación Universitaria Lasillista, Antioquía, Colombia. Julio-diciembre 2005: 50-63.

Center for Disease Control. Youth Violence: Fact Sheet. Us Department of Health and Human Services. 2007. En: <http://www. cdc.gov/ncipc/factsheets/yvfacts.htm> [Consultado: 2008].

Echeburúa, Enrique; Fernández-Montalvo, Javier y Corral, Paz. “Hay diferencias entre la violencia grave y la violencia menos grave contra la pareja?". International Journal of Clinical and Health Psychology 8 (2). Asociación Española de Psicología Conductual. Mayo 2008: 355-382.

Fritch, April M. \& Lynch, Shannon M. "Group treatment for adult survivors of interpersonal trauma”. Journal of Psychological Trauma 7 (3). Psychology Press Routledge Taylor \& Francis Group. September 2008: 145-169.

Glasser, William. Teoría de la elección, una nueva psicología de la libertad personal.
Barcelona. España: Ediciones Paidós Ibérica, SA, 1999.

Guenard Otero, Enitza Z. "Jóvenes víctima de violencia en la relación de pareja: Percepción de la violencia, patrones de crianza, escolarización y expectativas de roles en la relación de pareja en los jóvenes estudiantes de la Universidad de Puerto Rico, Recinto de Río Piedras, Humacao, Cayey y Utuado". [Tesis de maestría inédita]. Universidad de Puerto Rico, Recinto de Río Piedras, 1998.

Halpern, Carolyn T.; Young, Mary L.; Waller, Martha W.; Martin, Sandra L. y Kupper, Lawrence L. "Prevalence of partner violence in same-sex romantic and sexual relationships in a national simple of adolescents". Journal of Adolescent Health 35 (2). Journal for the Society of Adolescent Health and Medicine. August 2004: 124-131.

Jackson, Susan; Cram, Fiona y Seymour, Fred W. "Violence and sexual coercion in high school student's dating relationships". Journal of Family Violence 15 (1). Springer. March 2000: 23-36.

Kury, Helmut; Obergfell-Fuchs, Joachim y Woessner, Gunda. "The extent of family violence in Europe. A comparison of National Surveys". Violence Against Women 10 (7). Sage Journals. July 2004: 749-769.

Lizardi, María M. "Violencia doméstica: Un modelo grupal de intervención". Revista Análisis 3 (1). Escuela Graduada de Trabajo Social Universidad de Puerto Rico, Recinto de Río Piedras. 2002: 47-59.

Lizardi, María M. y Molina, Gloria M. "La intervención grupal como estrategia de cambio en situaciones de violencia familiar". Ponencia presentada en la Tercera Conferencia de Trabajo Social 
Forense, Oficina de Servicios Sociales de la Rama Judicial de Puerto Rico. 2003. En: <http://www.ramajudicial.pr/Miscel/ Conferencia/PDF/7_Lizardi_Molina.pdf> [Consultado: 2008].

Luccas Irizarry, Nydia \& Berríos Rivera, Reynaldo. Investigación cualitativa en educación y ciencias sociales. San Juan, PR: Publicaciones Puertorriqueñas, 2003.

Matud Aznar, María del Pilar. "Impacto de la violencia doméstica en la salud de la mujer maltratada". Psicothema 16 (3). Facultad de Psicología de la Universidad de Oviedo y el Colegio Oficial de Psicólogos del Principado de Asturias. 2004: 397-401.

Matud Aznar, María del Pilar; Gutiérrez, Ana Belén y Padilla, Vanessa. "Intervención psicológica con mujeres víctimas de maltrato por parte de su pareja". Papeles del Psicólogo 88. España: Consejo General de Colegios Oficiales de Psicólogos. Mayo 2004 [Revista Digital]. En: <http://www.papelesdelpsicologo.es/ vernumero.asp?id=1155> [Consultado: 2009].

Michigan Domestic Violence Prevention \& Treatment Board. "Violencia de pareja es un problema importante: Paquete educativo sobre la violencia en las parejas juveniles". 2006 [Revista Digital]. En: <http://www.michigan.gov/documents/ dhs/DHS-PUB-0224-SP_208541_7.pdf> [Consultado: 2007].

Muñoz Rivas, María Julia. "Violencia contra la mujer en las relaciones de noviazgo: causas, naturaleza y consecuencias". Universidad Autónoma de Madrid. 2006. [Revista Digital]. En: <http://www. inmujer.migualdad.es/mujer/mujeres/ estud_inves/Noviazgo.pdf > [Consultado: 2008].
Navarro Rodríguez, M. "Asertividad y Teoría de decisiones: El rol del orientador escolar". Durango, México. 2000. En: <http://www. alejandria.com/00/colaboraciones.htm> [Consultado: 2009].

Organización Mundial de la Salud. "Estudio pionero sobre la violencia doméstica". 2005. [Revista Digital]. En: <http://www. int/mediacentre/news/releases/2005/ pr62/es/index.html> [Consultado: 2008].

Partnership Against Domestic Violence. Rueda de Poder y Control. En: <http://www. padv.org/infostats.aspx > [Consultado: 2007].

Patton, Michael Quinn. Qualitative Evaluation and Research Methods. $2^{\text {nd }}$ ed. Thousand Oaks, CA: Sage Publications, 2002.

Rivera-Rivera, Leonor; Allen, Betania; Rodríguez Ortega, Graciela; ChávezAyala, Rubén y Lazcano-Ponce, Eduardo. "Violencia durante el noviazgo, depresión $y$ conducta de riesgo en estudiantes féminas (12-24 años)". Salud Pública de México 48 (2). Instituto Nacional de Salud Pública de México, 2006: 288-296.

Rodríguez Corvo, Dalia. "Noviazgo violento; caso ULA". Venezuela: Universidad de los Andes en la Ciudad de Mérida, 2007. [Revista Digital]. En: <http://www2.uacj. $\mathrm{mx} /$ universidadsaludable/memorias/ documentos/PDF\%20Resumenes/LA $\% 20$ EXPERIENCIA $\% 20$ DES DE $\% 20$ LA $\% 20 \% 20$ DIRECCI\%C3\%93N\%20DE $\% 20$ ASUNTOS\%20ESTUDIANTILES, $\% 20$ DAES. pdf> [Consultado: 2008].

Rogers, Carl. Psicoterapia centrada en el cliente. Barcelona: Paidós Ibérica, 1997.

Santiago, Esmeralda. El sueño de América. New York: Harper Libros, 1996. 
Strauss, Anselm. C. y Corbin, Juliet. Basics of qualitative research: Grounded theory procedures and techniques. Newbury Park, CA, EEUU: Sage, 1990.

UNICEF. "La violencia doméstica contra mujeres y niñas". Fondo de las Naciones Unidas para la Infancia. Centro de Investigaciones Innocenti de
UNICEF. Florencia, Italia. 2000. [Revista Digital] En: <http://www.unicef-irc. org/publications/pdf/digest6s.pdf > [Consultado: 2008].

Yalom, Irving D. Theory and Practice of Group Psychotherapy. $3^{\text {rd }}$ Edition. Basic Books, 1985. 